\title{
Vibration Control of Fractionally-Damped Beam Subjected to a Moving Vehicle and Attached to Fractionally-Damped Multiabsorbers
}

\author{
Hashem S. Alkhaldi, ${ }^{1}$ Ibrahim M. Abu-Alshaikh, ${ }^{1}$ and Anas N. Al-Rabadi ${ }^{2}$ \\ ${ }^{1}$ Mechanical Engineering Department, The University of Jordan, Amman 11942, Jordan \\ ${ }^{2}$ Computer Engineering Department, The University of Jordan, Amman 11942, Jordan \\ Correspondence should be addressed to Anas N. Al-Rabadi; a.alrabadi@ju.edu.jo
}

Received 8 September 2013; Accepted 6 November 2013

Academic Editor: Ming Li

Copyright ( 2013 Hashem S. Alkhaldi et al. This is an open access article distributed under the Creative Commons Attribution License, which permits unrestricted use, distribution, and reproduction in any medium, provided the original work is properly cited.

This paper presents the dynamic response of Bernoulli-Euler homogeneous isotropic fractionally-damped simply-supported beam. The beam is attached to multi single-degree-of-freedom (SDOF) fractionally-damped systems, and it is subjected to a vehicle moving with a constant velocity. The damping characteristics of the beam and SDOF systems are described in terms of fractional derivatives. Three coupled second-order fractional differential equations are produced and then they are solved by combining the Laplace transform with the decomposition method. The obtained numerical results show that the dynamic response decreases as (a) the number of absorbers attached to the beam increases and (b) the damping-ratios of used absorbers and beam increase. However, there are some critical values of fractional derivatives which are different from unity at which the beam has less dynamic response than that obtained for the full-order derivatives model. Furthermore, the obtained results show very good agreements with special case studies that were published in the literature.

\section{Introduction}

Many physical problems were adequately described by differential equations of fractional order. The list of these problems is long and the areas of applications are broad. Polymeric damping, fluid mechanics, and the theory of viscoelasticity [1-8] are some of these applications. The solution in the closed form to a class of SDOF fractional oscillators subjected to an impulse response is investigated [9]. Relationship between fractional oscillator processes and the corresponding fractional Brownian motion processes is well established in research. However, the increment process of fractional Brownian motion can be described by a random function with long-range dependence fractional Gaussian noise model [10]. A fractal time series is taken as the solution of differential equation of a fractional order, or a response of a fractional system, or a fractional filter driven with a white noise in the domain of stochastic processes [11, 12]. A general approach for approximating ideal filters that are based on fractional calculus from the point of view of systems of fractional order was introduced $[13,14]$. A new direct operational inversion method is introduced for solving coupled linear systems of ordinary fractional differential equations, where the obtained solutions are expressed explicitly in terms of multivariate Mittag-Leffler functions $[9,15,16]$. The solutions of fractional Langevin equation of two different orders which are known as the fractional Ornstein-Uhlenbeck processes, based on Weyl and Riemann-Liouville fractional derivatives were obtained $[9,17]$. Power-laws-type data may further, in earthquake and wind engineering applications, be governed by stochastically differential equations of fractional order [18, 19]. Analysis of signals generated by fractional-order process and applications of such model in a wide range of physical, mechanical, and biological systems are discussed in [20]. However, as noted from the literature, a clear physical interpretation of the fractional derivative has been elusive. Good examples are (1) transverse vibration of homogeneous beams whose damping behavior is described by a fractional derivative of predefined 
constant orders was an important subject of matter [11, 2124]; (2) diffusion process where fractional differential equations have been employed to describe an anomalous diffusion regime including both subdiffusion and superdiffusion [25]; and (3) many linear viscoelastic damping materials exhibit a macroscopic constitutive behavior which has been the subject of many investigations involving fractional order derivative [26]. However, in the fractional-derivative model of viscoelastic materials over extended ranges of time and frequency, the deformation work corresponding to springs and losses corresponding to dashpots (described by fractional derivatives) have both energy types (stored and dissipated) at any point of the utilized materials $[27,28]$.

The dynamic response characteristics of beams resulting from the passage of different moving load systems, and with the existence of dampers and oscillators, have been studied within many applications. Some of the applications consist of the vibrations existing in bridges, aircraft carriers, and railroad tracks due to moving vehicles, and vibrations existing in pipeline systems due to fluid flows [29-31]. One of the main objectives of studying such problems is to minimize or to suppress the dynamic response characteristics or vibration levels of beams when subjected to different load systems. The simplest and most economical way to achieve this objective is by having different lumped appendages attached to the beam at different locations. In investigating the dynamic response of beams, if the travelling force is constant, then the time-dependent coefficients can be obtained in analytical forms [32] which make it easy to find a solution to any particular problem. However, in many cases, it is not just the response of the beam (which is a function of the spatial variable and time and depends on several parameters such as beam characteristics, the magnitude of the force, its velocity, number of appendages attached to the beam, and their locations) that the designer is interested in. However, it has long been observed that, as a structure is subjected to moving loads, the induced dynamic deflection and stress can be significantly higher than those observed in the static case [33]. In this regard, the majority of the literature has been devoted to the study of the so-called moving force [34], moving mass [35-38], moving oscillator problems [39-41], and multiple moving oscillators [42].

The vibration of a beam excited by a moving unmaintained oscillator is discussed in [43]. In this study, for certain values of system parameters and travelling speeds of the moving oscillator, separation of the oscillator from the beam can occur frequently and even more than once during the travel of the load [43]. The problem of an oscillator traversing on an elastically-supported continuum is studied, whereas the flexibility in the boundaries of the continuum is modeled by linear-transverse springs [33]. Green functions formulation to analyze the free vibration of a linear undamped beam subjected to grounded SDOF oscillators appendages at different discrete points is used [44], where researchers compared their results with the approximate solution obtained by Galarkin's method.

Searching the relevant literature shows only few papers dealing with the vibration of beams with constant values for the fractional damping behavior [23, 24, 45]. However, in this study, the fractional damping behaviors of the beam and absorbers are taken with arbitrary orders. Therefore, this study is important for the following key reasons: the damping behavior of the dynamic response of the beam can be analyzed over a continuous wide range by means of fractional-derivative parameters. Moreover, the fractional derivative damping behavior may better converge to realistic experimental models than the well-known first order damping model. Hence, the fractional derivative model may precisely prescribe a nonlinear damping behavior than other existing damping models.

In this paper, a homogeneous isotropic Euler beam with different appendages attached at different locations subjected to a uniformly moving vehicle is investigated. The solution to the handled problem is based on decomposing the generalized transverse displacements of the beam, attached oscillators, and the moving vehicle into infinite series components. Then, the generalized displacements are recursively solved by in the Laplace transform domain. The solutions in this work mainly focus on the following characteristics: the maximum response of the beam for a given velocity of the vehicle, the maximum response over all possible velocities and the velocity at which it occurs, the optimal beam and absorbers fractional-damping characteristics, and the effect of the number of attached absorbers.

\section{Formulation of the Problem}

Transverse vibration of Bernoulli-Euler homogeneous isotropic fractionally-damped simply-supported beam is investigated. The beam is assumed to be subjected to a vehicle moving from left-to-right with a constant velocity $(v)$. The governing equation of the beam under investigation, whose damping characteristics are described by a fractional derivative of order $\beta$, can be written as

$$
\begin{gathered}
E I \frac{\partial^{4} w(x, t)}{\partial x^{4}}+C \frac{\partial^{\beta} w(x, t)}{\partial t^{\beta}}+\mu \frac{\partial^{2} w(x, t)}{\partial t^{2}} \\
=-m_{v}(\ddot{q}-g) \delta(x-v t) H\left(\frac{L}{v}-t\right) \\
\quad-\sum_{i=1}^{N} \widehat{M}_{i} \widehat{\ddot{z}}_{i}(t) \delta\left(x-d_{i}\right), \quad x \in(0, L), t>0, \\
\text { BC's: } w(0, t)=0, \quad w(L, t)=0, \quad w_{x x}(0, t)=0, \\
\quad w_{x x}(L, t)=0, \quad \frac{d w(x, 0)}{d t}=0,
\end{gathered}
$$

where $H(\cdot)$ refers to the unit step Heaviside function and $\delta(\cdot)$ refers to the Kronecker delta function. The equations corresponding to the $N$-SDOF absorbers that are appendaged to the beam are given as

$$
\begin{aligned}
\widehat{M}_{i} \widehat{\ddot{z}}_{i}(t)= & \widehat{K}_{i}\left[w\left(d_{i}, t\right)-\widehat{z}_{i}(t)\right] \\
& +\widehat{C}_{i} \frac{d^{\gamma}\left[w\left(d_{i}, t\right)-\widehat{z}_{i}(t)\right]}{d t^{\gamma}}, \quad t>0,
\end{aligned}
$$




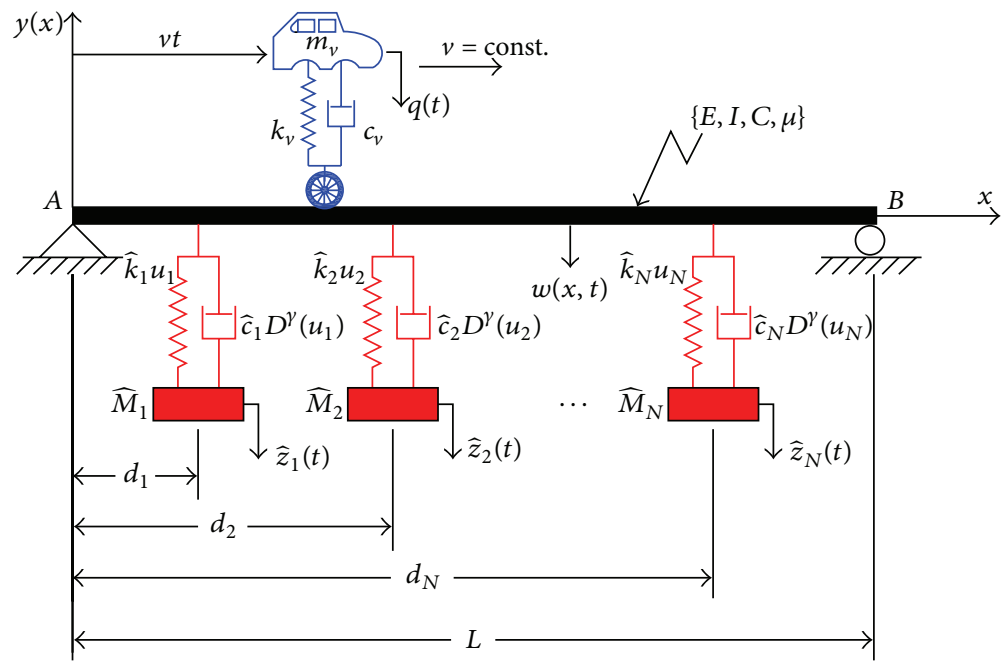

FIGURE 1: A Simply-supported beam attached to $N$-absorbers and subjected to a moving vehicle with a constant speed.

$$
\text { IC's: } \widehat{z}_{i}(0)=0, \quad \frac{d \widehat{z}_{i}(0)}{d t}=0 .
$$

The equations corresponding to the moving vehicle can be expressed as

$$
\begin{aligned}
& m_{v}(\ddot{q}(t)-g)=k_{v}[w(v t, t)-q(t)] \\
& \quad+c_{v} \frac{d[w(v t, t)-q(t)]}{d t}, \quad 0<t<\frac{v}{L}, \\
& \text { IC's: } q(0)=0, \quad \frac{d q(0)}{d t}=0,
\end{aligned}
$$

where the beam is assumed to be initially at rest and $\{L, E, I, \mu, C\}$ are the length, modulus of elasticity, moment of inertia of cross-sectional area $(A)$, mass per unit length, and the coefficient of external damping of the beam, respectively. Furthermore, these values are assumed to be constants. The $N$-rigid-body SDOF fractionally-damped absorbers of order $\gamma$, masses $\widehat{M}_{i}$, stiffnesses $\widehat{K}_{i}$, and damping constants $\widehat{C}_{i}$ are attached to the beam, as shown in Figure 1 (for $i=1,2, \ldots, N)$. These elements are located at distances $d_{i}$ measured from the left-end of the beam. However, the parameters $\left\{m_{v}, k_{v}, c_{v}\right\}$ refer to mass, stiffness, and damping constants of the vehicle, respectively. Furthermore, the set $\left\{w(x, t), \widehat{z}_{i}(t), q(t)\right\}$ represents the beam transverse deflection at location $(x)$ and time $(t)$, the transverse dynamic displacements of the masses $\widehat{M}_{i}$, and vehicle's transverse displacement, respectively.

Equations that are similar to (1a), (1b), (1c), (2a), (2b), (3a), and (3b) are presented in [42], but with the conventional first order derivative damping behaviors. The fractional derivatives of order $\beta$ or $\gamma$, presented in (1a) and (2a), may be defined in many ways [47]. Among these are the two most frequently encountered, the Riemann-Liouville and Caputo fractional derivatives of order $\beta$ which are defined by the following convolution integrals, respectively:

$$
\frac{\partial^{\beta} w(x, t)}{\partial t^{\beta}}=\frac{1}{\Gamma(k-\beta)} \frac{\partial^{k}}{\partial t^{k}} \int_{0}^{t} \frac{w(x, u)}{(t-u)^{\beta+1-k}} d u,
$$

$$
\frac{\partial^{\beta} w(x, t)}{\partial t^{\beta}}=\frac{1}{\Gamma(k-\beta)} \int_{0}^{t} \frac{1}{(t-u)^{\beta+1-k}} \frac{\partial^{k} w(x, u)}{\partial u^{k}} d u,
$$

where $k$ is a positive integer such that $(k-1 \leq \beta<k)$.

In modal form, the transverse deflection of the simplysupported beam can be written as $[31,32]$ :

$$
w(x, t)=\sum_{n=1}^{\infty} Y_{n}(t) X_{n}(x)
$$

where $Y_{n}(t)$ is the generalized displacement or the modal response of the beam and $X_{n}(x)$ are the normal modes of the undamped free vibration of the simply-supported beam written as [46]

$$
X_{n}(x)=\sin \left(\kappa_{n} x\right)
$$

where $\left(\kappa_{n}=n \pi / L\right)$ is the frequency parameter associated with the simply-supported beam. Equation (6) should satisfy the following fourth-order partial differential equation:

$$
E I \frac{\partial^{4} X_{n}(x)}{\partial x^{4}} Y_{n}(t)+\mu \frac{\partial^{2} Y_{n}(t)}{\partial t^{2}} X_{n}(x)=0
$$

Substituting (5) into (1a), (2a), and (3a) leads to the following coupled-system of fractional differential equations:

$$
\begin{gathered}
E I \sum_{n=1}^{\infty} Y_{n}(t) \frac{d^{4} X_{n}(x)}{d x^{4}}+C \sum_{n=1}^{\infty} \frac{d^{\beta} Y_{n}(t)}{d t^{\beta}} X_{n}(x) \\
\quad+\mu \sum_{n=1}^{\infty} \frac{d^{2} Y_{n}(t)}{d t^{2}} X_{n}(x) \\
=-m_{v}(\ddot{q}-g) \delta(x-v t) H\left(\frac{L}{v}-t\right) \\
\quad-\sum_{i=1}^{N} \widehat{M}_{i} \widehat{\ddot{z}}_{i}(t) \delta\left(x-d_{i}\right),
\end{gathered}
$$




$$
\begin{aligned}
\widehat{M}_{i} \widehat{\ddot{z}}_{i}(t)= & -\left(\widehat{K}_{i} \widehat{z}_{i}(t)+\widehat{C}_{i} \frac{d^{\gamma} \widehat{z}_{i}(t)}{d t^{\gamma}}\right) \\
& +\sum_{r=1}^{\infty}\left(\widehat{K}_{i} Y_{r}(t) X_{r}\left(d_{i}\right)+\widehat{C}_{i} \frac{d^{\gamma} Y_{r}(t)}{d t^{\gamma}} X_{r}\left(d_{i}\right)\right), \\
m_{v} \ddot{q}(t)= & m_{v} g-\left(k_{v} q(t)+c_{v} \frac{d q(t)}{d t}\right) \\
& +\sum_{p=1}^{\infty}\left(k_{v} Y_{p}(t) X_{p}(v t)+c_{v} \frac{d Y_{p}(t)}{d t} X_{p}(v t)\right) .
\end{aligned}
$$

Multiplying (8a) by $X_{m}(x)$ and integrating over the domain $(0 \leq x \leq L)$ yields

$$
\begin{array}{rl}
\sum_{n=1}^{\infty} \int_{0}^{L} & E I Y_{n}(t) \frac{d^{4} X_{n}(x)}{d x^{4}} X_{m}(x) d x \\
& +\sum_{n=1}^{\infty} \int_{0}^{L} \frac{d^{\beta} Y_{n}(t)}{d t^{\beta}} C X_{n}(x) X_{m}(x) d x \\
& +\sum_{n=1}^{\infty} \int_{0}^{L} \frac{d^{2} Y_{n}(t)}{d t^{2}} \mu X_{n}(x) X_{m}(x) d x \\
= & -\int_{0}^{L} m_{v}(\ddot{q}-g) X_{m}(x) \delta(x-v t) H\left(\frac{L}{v}-t\right) d x \\
& -\sum_{i=1}^{N} \int_{0}^{L} \widehat{M}_{i} \widehat{z}_{i}(t) X_{m}(x) \delta\left(x-d_{i}\right) d x .
\end{array}
$$

The dynamic response of the beam represented by (1a) is analyzed after projecting the 4th order partial derivative into a complete orthogonal basis. For the present problem, the eigenfunctions of the linear operator representing the simply-supported beam without appendages given in (6) can be used. These eigenfunctions should satisfy the following orthogonality conditions $[32,42]$ :

$$
\begin{array}{r}
\int_{0}^{L} \mu X_{n}(x) X_{m}(x) d x=\frac{\mu L}{2} \delta_{n m}, \\
\int_{0}^{L}\left(E I X_{n}^{\prime \prime}(x)\right)^{\prime \prime} X_{m}(x) d x=\frac{E I n^{4} \pi^{4}}{2 L^{3}} \delta_{n m}, \\
\text { for } n, m=1,2,3, \ldots
\end{array}
$$

By considering these orthogonality conditions, the differential equation of the $n$th mode of the generalized beamdisplacement, (9), can be rewritten as

$$
\begin{aligned}
\sum_{n=1}^{\infty} Y_{n}(t) & \frac{E n^{4} \pi^{4}}{2 L^{3}} \delta_{n m}+\sum_{n=1}^{\infty} \frac{d^{\beta} Y_{n}(t)}{d t^{\beta}} \frac{C L}{2} \delta_{n m} \\
& +\sum_{n=1}^{\infty} \frac{d^{2} Y_{n}(t)}{d t^{2}} \frac{\mu L}{2} \delta_{n m} \\
= & -m_{v}(\ddot{q}-g) X_{m}(v t) H\left(\frac{L}{v}-t\right)-\sum_{i=1}^{N} \widehat{M}_{i} \widehat{\ddot{z}}_{i}(t) X_{m}\left(d_{i}\right) .
\end{aligned}
$$

Substituting (6) into (8a), (8b), (8c), and (11) and noting that $(m=n)$ lead to the following coupled system of fractional differential equations:

$$
\begin{gathered}
\frac{d^{2} Y_{n}(t)}{d t^{2}}+2 \omega_{n} \zeta_{n} \frac{d^{\beta} Y_{n}(t)}{d t^{\beta}}+\omega_{n}^{2} Y_{n}(t) \\
=-\eta_{1}\left(m_{v}(\ddot{q}-g) \sin \left(\Omega_{n} t\right) H\left(\frac{L}{v}-t\right)\right. \\
\left.+\sum_{i=1}^{N} \widehat{M}_{i} \hat{z}_{i}(t) \sin \left(\frac{\Omega_{n} d_{i}}{v}\right)\right), \\
\widehat{\ddot{z}}_{i}(t)=-\left(\widehat{\omega}_{i}^{2} \widehat{z}_{i}(t)+2 \widehat{\omega}_{i} \widehat{\zeta}_{i} \frac{d^{\gamma} \widehat{z}_{i}(t)}{d t^{\gamma}}\right) \\
+\sum_{r=1}^{\infty}\left[\widehat{\omega}_{i}^{2} Y_{r}(t) \sin \left(\frac{\Omega_{r} d_{i}}{v}\right)\right. \\
\left.+\frac{d^{\gamma} Y_{r}(t)}{d t{ }^{\gamma}} 2 \widehat{\omega}_{i} \widehat{\zeta}_{i} \sin \left(\frac{\Omega_{r} d_{i}}{v}\right)\right], \\
\ddot{q}(t)=g-\left(\omega_{v}^{2} q(t)+2 \omega_{v} \zeta_{v} \frac{d q(t)}{d t}\right) \\
+\sum_{p=1}^{\infty}\left(\omega_{v}^{2} Y_{p}(t) \sin \left(\Omega_{p} t\right)\right. \\
\left.+2 \omega_{v} \zeta_{v} \frac{d Y_{p}(t)}{d t} \sin \left(\Omega_{p} t\right)\right),
\end{gathered}
$$

where

$$
\begin{gathered}
\omega_{n}=\kappa_{n}^{2} \sqrt{\frac{E I}{\mu}}, \\
\zeta_{n}=\frac{C}{2 \mu \omega_{n}}, \\
\widehat{\zeta}_{i}=\frac{\widehat{C}_{i}}{2 \widehat{M}_{i} \omega_{i}}, \\
\zeta_{v}=\frac{c_{v}}{2 m_{v} \omega_{v}}, \\
\omega_{v}=\sqrt{\frac{k_{v}}{m_{v}}} \\
\widehat{\omega}_{i}=\sqrt{\frac{\widehat{K}_{i}}{\widehat{M}_{i}}} \\
\Omega_{n}=\frac{n \pi v}{L}
\end{gathered}
$$

are the undamped natural circular frequency $\left(\omega_{n}\right)$, beam damping ratio $\left(\zeta_{n}\right)$, ith oscillator damping ratio $\left(\widehat{\zeta}_{i}\right)$, vehicle damping ratio $\left(\zeta_{v}\right)$, the natural frequency of the vehicle $\left(\omega_{v}\right)$, the natural frequency of the $i$ th oscillator $\left(\widehat{\omega}_{i}\right)$, and the load frequency $\left(\Omega_{n}\right)$, respectively, where $\left(\eta_{1}=2 / \mu L\right)$. 
To find the solution of the system in (12a), (12b), and (12c), associated with the boundary and initial conditions given in (1b), (1c), (2b), and (3b), the dependent variables $Y_{n}(t), \widehat{z}_{i}(t)$, and $q(t)$ can be decomposed into the following infinite series:

$$
\begin{aligned}
Y_{n}(t) & =\sum_{k=0}^{\infty} Y_{n}^{k}(t)=Y_{n}^{0}(t)+Y_{n}^{1}(t)+Y_{n}^{2}(t)+\cdots \\
\widehat{z}_{i}(t) & =\sum_{k=0}^{\infty} \widehat{z}_{i}^{k}(t)=\widehat{z}_{i}^{0}(t)+\widehat{z}_{i}^{1}(t)+\widehat{z}_{i}^{2}(t)+\cdots \\
q(t) & =\sum_{k=0}^{\infty} q^{k}(t)=q^{0}(t)+q^{1}(t)+q^{2}(t)+\cdots
\end{aligned}
$$

where $Y_{n}^{k}(t), \widehat{z}_{i}^{k}(t)$, and $q^{k}(t)$ for $(k=0,1,2, \ldots)$ are the components of $Y_{n}(t), \widehat{z}_{i}(t)$, and $q(t)$, respectively. The components of $Y_{n}(t), \widehat{z}_{i}(t)$, and $q(t)$ can be determined recursively, by substituting (14a), (14b), and (14c) into (12a), $(12 \mathrm{~b})$, and (12c), from the following equations:

$$
\begin{aligned}
& \frac{d^{2} Y_{n}^{0}(t)}{d t^{2}}+\omega_{n}^{2} Y_{n}^{0}(t)=\eta_{1} m_{v} g \sin \left(\Omega_{n} t\right) H\left(\frac{L}{v}-t\right), \\
& \frac{d^{2} z_{i}^{0}(t)}{d t^{2}}=\sum_{r=1}^{\infty} \sin \left(\frac{\Omega_{r} d_{i}}{v}\right)\left[\widehat{\omega}_{i}^{2} Y_{r}^{0}(t)+\frac{d^{\gamma} Y_{r}^{0}(t)}{d t^{\gamma}} 2 \widehat{\omega}_{i} \widehat{\zeta}_{i}\right],
\end{aligned}
$$

$$
\begin{gathered}
\frac{d^{2} q^{0}(t)}{d t^{2}}=g+\sum_{p=1}^{\infty} \sin \left(\Omega_{p} t\right)\left(\omega_{v}^{2} Y_{p}^{0}(t)+2 \omega_{v} \zeta_{v} \frac{d Y_{p}^{0}(t)}{d t}\right) \\
\frac{d^{2} Y_{n}^{k+1}(t)}{d t^{2}}+\omega_{n}^{2} Y_{n}^{k+1}(t) \\
=-2 \omega_{n} \zeta_{n} \frac{d^{\beta} Y_{n}^{k}(t)}{d t^{\beta}} \\
-\eta_{1}\left(m_{v} \frac{d^{2} q^{k}(t)}{d t^{2}} \sin \left(\Omega_{n} t\right) H\left(\frac{L}{v}-t\right)\right. \\
\left.\quad+\sum_{i=1}^{N} \widehat{M}_{i} \frac{d^{2} z_{i}^{k}(t)}{d t^{2}} \sin \left(\frac{\Omega_{n} d_{i}}{v}\right)\right),
\end{gathered}
$$

$$
\begin{aligned}
& \frac{d^{2} z_{i}^{k+1}(t)}{d t^{2}} \\
& =-\left(\widehat{\omega}_{i}^{2} z_{n}^{k}(t)+2 \widehat{\omega}_{i} \widehat{\zeta}_{i} \frac{d^{\gamma} z_{n}^{k}(t)}{d t^{\gamma}}\right) \\
& \quad+\sum_{r=1}^{\infty} \sin \left(\frac{\Omega_{r} d_{i}}{v}\right)\left[\widehat{\omega}_{i}^{2} Y_{r}^{k+1}(t)+\frac{d^{\gamma} Y_{r}^{k+1}(t)}{d t^{\gamma}} 2 \widehat{\omega}_{i} \widehat{\zeta}_{i}\right],
\end{aligned}
$$

$$
\begin{aligned}
& \frac{d^{2} q^{k+1}(t)}{d t^{2}} \\
& =-\left(\omega_{v}^{2} q^{k}(t)+2 \omega_{v} \zeta_{v} \frac{d q^{k}(t)}{d t}\right) \\
& \quad+\sum_{p=1}^{\infty} \sin \left(\Omega_{p} t\right)\left(\omega_{v}^{2} Y_{p}^{k+1}(t)+2 \omega_{v} \zeta_{v} \frac{d Y_{p}^{k+1}(t)}{d t}\right),
\end{aligned}
$$

for $k \geq 0$. Now, using the Laplace transform for the Caputo fractional derivative which is defined in (4b) as [47]

$$
\begin{aligned}
\ell\left(\frac{d^{\beta} Y_{n}^{k}(t)}{d t^{\beta}}\right)=s^{\beta} y_{n}^{k}(s)- & \sum_{j=0}^{m-1} s^{\beta-j-1}\left(y_{n}^{k}\right)^{j}(0), \\
& \text { for } m-1<\beta \leq m,
\end{aligned}
$$

the Laplace transforms of (14a), (14b), (14c), (15a), (15b), (15c), (15d), (15e), and (15f), with the related homogeneous initial conditions, can be rewritten as

$$
\begin{aligned}
& y_{n}(s)=\sum_{k=0}^{\infty} y_{n}^{k}(s), \\
& Z_{i}(s)=\sum_{k=0}^{\infty} Z_{i}^{k}(s), \\
& Q(s)=\sum_{k=0}^{\infty} Q^{k}(s),
\end{aligned}
$$

where

$$
\begin{gathered}
y_{n}^{0}(s)=\frac{\eta_{1} m_{v} g}{\left(s^{2}+\omega_{n}^{2}\right)} \ell\left(\sin \left(\Omega_{n} t\right) H\left(\frac{L}{v}-t\right)\right), \\
Z_{i}^{0}(s)=\sum_{r=1}^{\infty} \sin \left(\frac{\Omega_{r} d_{i}}{v}\right)\left(\frac{\widehat{\omega}_{i}^{2}+2 \widehat{\omega}_{i} \widehat{\zeta}_{i} s^{\gamma}}{s^{2}}\right) y_{r}^{0}(s), \quad(18 \mathrm{~b}) \\
Q^{0}(s)=\frac{g}{s^{3}}+\sum_{p=1}^{\infty}\left(\frac{\omega_{v}^{2} \ell\left(\sin \left(\Omega_{p} t\right) Y_{p}^{0}(t)\right)}{s^{2}}\right) \\
\left.+\frac{2 \omega_{v} \zeta_{v} \ell\left(\sin \left(\Omega_{p} t\right)\left(d Y_{p}^{0}(t) / d t\right)\right)}{s^{2}}\right), \\
y_{n}^{k+1}(s) \\
=-\frac{2 \omega_{n} \zeta_{n} s^{\beta}}{\left(s^{2}+\omega_{n}^{2}\right)} \\
-\eta_{1}\left(\frac{m_{v} \ell\left(d^{2} q^{k}(t) / d t^{2} \sin \left(\Omega_{n} t\right) H(L / v-t)\right)}{\left(s^{2}+\omega_{n}^{2}\right)}\right. \\
\left.+\sum_{i=1}^{N} \frac{\widehat{M}_{i} s^{2} Z_{i}^{k}(s) \sin \left(\Omega_{n} d_{i} / v\right)}{\left(s^{2}+\omega_{n}^{2}\right)}\right),
\end{gathered}
$$




$$
\begin{aligned}
Z_{i}^{k+1}(s)= & -\left(\widehat{\omega}_{i}^{2} \frac{Z_{i}^{k}(s)}{s^{2}}+2 \widehat{\omega}_{i} \widehat{\zeta}_{i} \frac{s^{\gamma} Z_{i}^{k}(s)}{s^{2}}\right) \\
& +\sum_{r=1}^{\infty} \sin \left(\frac{\Omega_{r} d_{i}}{v}\right)\left[\widehat{\omega}_{i}^{2} \frac{y_{r}^{k+1}(s)}{s^{2}}\right. \\
& \left.+2 \widehat{\omega}_{i} \widehat{\zeta}_{i} \frac{s^{\gamma} y_{r}^{k+1}(s)}{s^{2}}\right], \\
Q^{k+1}(s)=- & \frac{\omega_{v}^{2} Q^{k}(s)}{\left.s^{2}+\frac{2 s Q^{k}(s) \omega_{v} \zeta_{v}}{s^{2}}\right)} \\
+ & \sum_{p=1}^{\infty}\left(\frac{\omega_{v}^{2} \ell\left(\sin \left(\Omega_{p} t\right) Y_{p}^{k+1}(t)\right)}{s^{2}}\right. \\
& \left.+\frac{2 \omega_{v} \zeta_{v} \ell\left(\sin \left(\Omega_{p} t\right)\left(d Y_{p}^{k+1}(t) / d t\right)\right)}{s^{2}}\right),
\end{aligned}
$$

for $k \geq 0$ and $(i=1,2, \ldots, N)$, where $N$ is the number of the SDOF fractionally-damped absorbers attached to the beam. In the above equations $\ell(\cdot)$ designates the Laplace transform of $(\cdot)$ where $\left(\ell\left(\widehat{z}_{i}(t)\right)=Z_{i}(s), \ell\left(Y_{n}(t)\right)=y_{n}(s)\right.$ and $(\ell(q(t))=$ $Q(s)$. Nevertheless, the explicit forms of (18a), (18b), (18c), $(18 \mathrm{~d})$, and (18e) for $k \geq 0$ are rather long and complex. However, when the damping effect is fractional, these equations contain the well-known hepergeometric function defined as [48]

$$
\begin{aligned}
& \text { hypergeom }\left(\left[n_{1}\right],\left[d_{1}, d_{2}\right], z\right) \\
& =\sum_{k=0}^{\infty} \frac{z^{k}\left(\Gamma\left(n_{1}+k\right) / \Gamma\left(n_{1}\right)\right)}{k !\left(\prod_{j=1}^{2}\left(\Gamma\left(d_{j}+k\right) / \Gamma\left(d_{j}\right)\right)\right)} .
\end{aligned}
$$

The term-by-term inversion of (18a), (18b), (18c), (18d), and (18e) with (14a), (14b), and (14c) represents the corresponding solution of the handled problem. However, many problems can be treated as special cases of (18a), (18b), (18c), (18d), and (18e); the absorbers can be neglected by assuming that $\left(\widehat{\omega}_{i}=0\right)$ and the moving vehicle can be treated as a moving load by assuming $\left(\zeta_{v}=1\right.$ and $\left.k_{v}=1\right)$ or by replacing the Heaviside step function by unity, where (in this case) the gravity acceleration $(g)$ is further replaced by the intensity of the moving load.

\section{Results and Discussion}

In this Section, two examples are given to verify the validity of the formal technique used in this paper. Moreover, three more important case studies are introduced to show the effect of fractional-damping behaviors for both of the beam and the SDOF absorbers and the effect of the number of SDOF absorbers attached to the beam $(N)$. In all case studies, the dynamic response of the beam is mainly governed by the fundamental mode $(n=1)$ of the beam because this mode has the significant effect on the beam dynamic response.

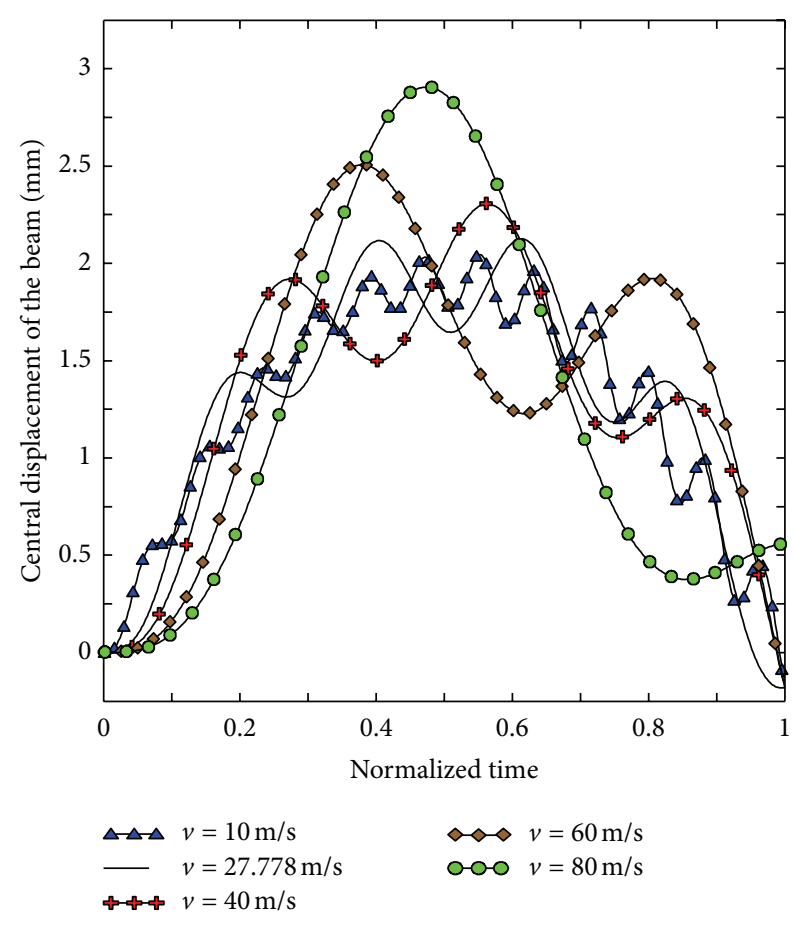

FIGURE 2: The dynamic response of an elastic beam at mid-span for many load speeds.

3.1. Verification Problem I. This example verifies the validity of the technique used in this paper. In this example, an undamped beam with a moving oscillator is investigated [49]. The parameters adopted for the beam under investigation are given in Table 1. The displacement response for the beam versus the normalized time is shown in Figure 2; that is, when $(t=0)$ the load enters the beam from left-hand side $(x=0)$ and when $(t=L / v)$ the moving load system leaves the righthand side of the beam $(x=L)$. The curves of Figure 2 show the dynamic mid-span displacement response of the beam for the five cases of traveling velocities: $v=10 \mathrm{~m} / \mathrm{s}, v=27.778 \mathrm{~m} / \mathrm{s}$, $v=40 \mathrm{~m} / \mathrm{s}, v=60 \mathrm{~m} / \mathrm{s}$, and $v=80 \mathrm{~m} / \mathrm{s}$, respectively.

In this example, it is shown that the dynamic displacement response increases as the traveling speed increase. This follows from the fact that the energy content of the elastic beam increases when the speed increases. Furthermore, Figure 2 shows that the amplitude of the displacement response for the force and mass moving systems increases as speed increases. The maximum displacement when $v=$ $27.778 \mathrm{~m} / \mathrm{s}$ is $\left(w_{\max }=2.17453 \mathrm{~m} / \mathrm{s}\right)$ which satisfies the results obtained by Duhamel integral [32] and is almost the same as that presented in [49].

3.2. Verification Problem II. This verification problem presents a damped beam attached to a single absorber and subjected to a moving load of intensity $9.81 \mathrm{~N}$; that is, the moving vehicle of unit mass approaches to the moving load $\left(k_{v}=0 \mathrm{~N} / \mathrm{m}, \zeta_{v}=1 \mathrm{Ns} / \mathrm{m}, m_{v}=1 \mathrm{~kg}\right)$. The data presented in $[50,51]$ are used for solving this problem, see Table 1. In this problem, the response of an initially stationary beam subjected to a moving load with a constant velocity is 
TABLE 1: Material and geometrical properties of beam, vehicle, and absorbers.

\begin{tabular}{|c|c|}
\hline Problem & Properties \\
\hline $\begin{array}{l}\text { Verification problem I in } \\
\text { Section } 3.1\end{array}$ & $\begin{array}{c}L=25 \mathrm{~m}, E=82.7 \mathrm{GPa}, I=9.2 \mathrm{~m}^{4}, \mu=2303 \mathrm{~kg} / \mathrm{m}, m_{v}=5750 \mathrm{~kg}, k_{v}=1595000 \mathrm{~N} / \mathrm{m}, c_{v}=0 \mathrm{Ns} / \mathrm{m}, \\
\widehat{K}_{i}=0 \mathrm{~N} / \mathrm{m}, \widehat{\omega}_{i}=0 \mathrm{rad} / \mathrm{s} .\end{array}$ \\
\hline $\begin{array}{l}\text { Verification problem II in } \\
\text { Section } 3.2\end{array}$ & $\begin{array}{c}\beta=1, \gamma=1, N=1:\left\{d_{1}=L / 2(\mathrm{~m})\right\},(i=1,2, \ldots, N) \\
N=2:\left\{\begin{array}{c}d_{1}=\left(\frac{L}{2}-\delta\right) \mathrm{m} \\
d_{2}=\left(\frac{L}{2}+\delta\right) \mathrm{m}\end{array}\right\}, \quad N=3:\left\{\begin{array}{c}d_{1}=\left(\frac{L}{2}-\delta\right) \mathrm{m} \\
d_{2}=\left(\frac{L}{2}\right) \mathrm{m} \\
d_{3}=\left(\frac{L}{2}+\delta\right) \mathrm{m}\end{array}\right\}, \quad \delta=0.02 \mathrm{~m} \\
k_{v}=0.0 \mathrm{~N} / \mathrm{m}, \zeta_{v}=0.1, m_{v}=1 \mathrm{~kg}\end{array}$ \\
\hline Problem in Section 3.4 & $N=1, d_{1}=(L / 2) \mathrm{m}, v=25 \mathrm{~m} / \mathrm{s}, m_{v}=1 \mathrm{~kg}, k_{v}=700 \mathrm{~N} / \mathrm{m}, \zeta_{v}=0.1, \zeta=0.15$ \\
\hline Problem in Section 3.5 & $\begin{array}{c}\beta=1.245, \gamma=1.19, k_{v}=700.0 \mathrm{~N} / \mathrm{m}, \zeta_{v}=0.1, m_{v}=1 \mathrm{~kg} \\
N=1:\left\{d_{1}=\frac{L}{2}(\mathrm{~m})\right\}, \quad N=2:\left\{\begin{array}{c}d_{1}=\left(\frac{L}{2}-\delta\right) \mathrm{m} \\
d_{2}=\left(\frac{L}{2}+\delta\right) \mathrm{m}\end{array}\right\} \\
N=3:\left\{\begin{array}{c}d_{1}=\left(\frac{L}{2}-\delta\right) \mathrm{m} \\
d_{2}=\left(\frac{L}{2}\right) \mathrm{m} \\
d_{3}=\left(\frac{L}{2}+\delta\right) \mathrm{m}\end{array}\right\}, \quad \delta=0.02 \mathrm{~m}\end{array}$ \\
\hline $\begin{array}{l}\text { For all problems (except } \\
\text { verification Problem I) }\end{array}$ & $\begin{array}{c}L=4 \mathrm{~m}, E=206.8 \mathrm{GPa}, \rho=7820 \mathrm{~kg} / \mathrm{m}^{3}, A=0.03 \times 0.03 \mathrm{~m}^{2}, \widehat{M}_{i}=1.4076 \mathrm{~kg}, \widehat{K}_{i}=877.767 \mathrm{~N} / \mathrm{m}, \\
\widehat{\zeta}_{i}=0.1846356 \text { for } i=1,2, \ldots, N .\end{array}$ \\
\hline
\end{tabular}

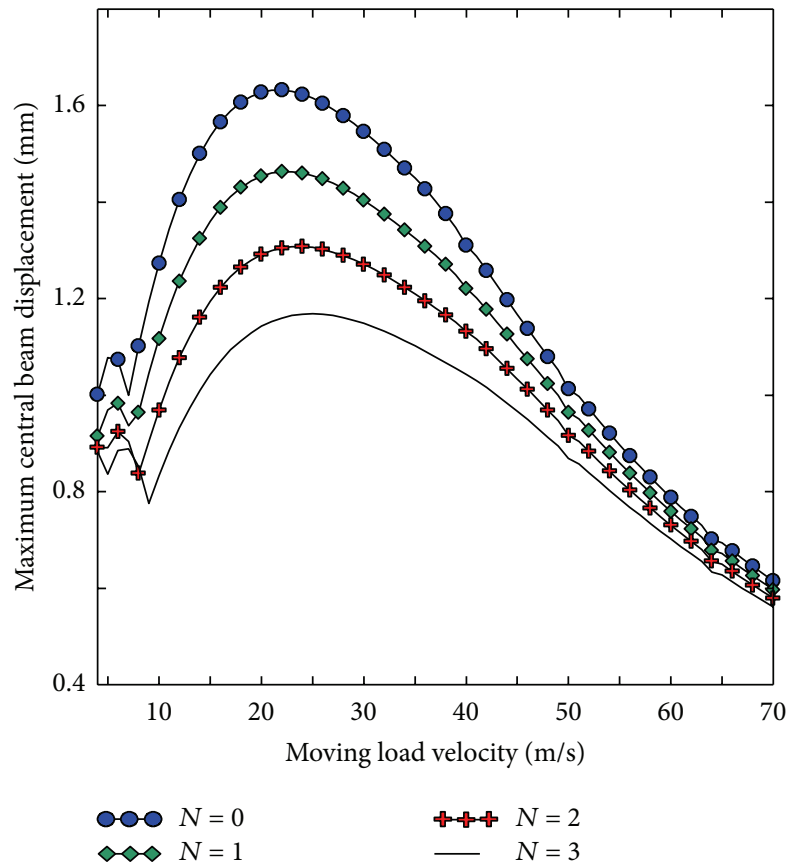

FIGURE 3: The maximum displacement of the beam at mid-span versus the velocity of the moving load for $\zeta=0$.

considered. The beam is assumed to be free or appendaged to one, two, or three absorbers $(N=0,1,2,3)$ and $(i=1, \ldots, N)$ at different locations of the beam, see Figures 3,4 , and 5.

In this problem, $(N=0)$ refers to a beam without any appendages; that is, all parametric properties of the oscillators are null. The case for $(N=1)$ was studied in [51] as

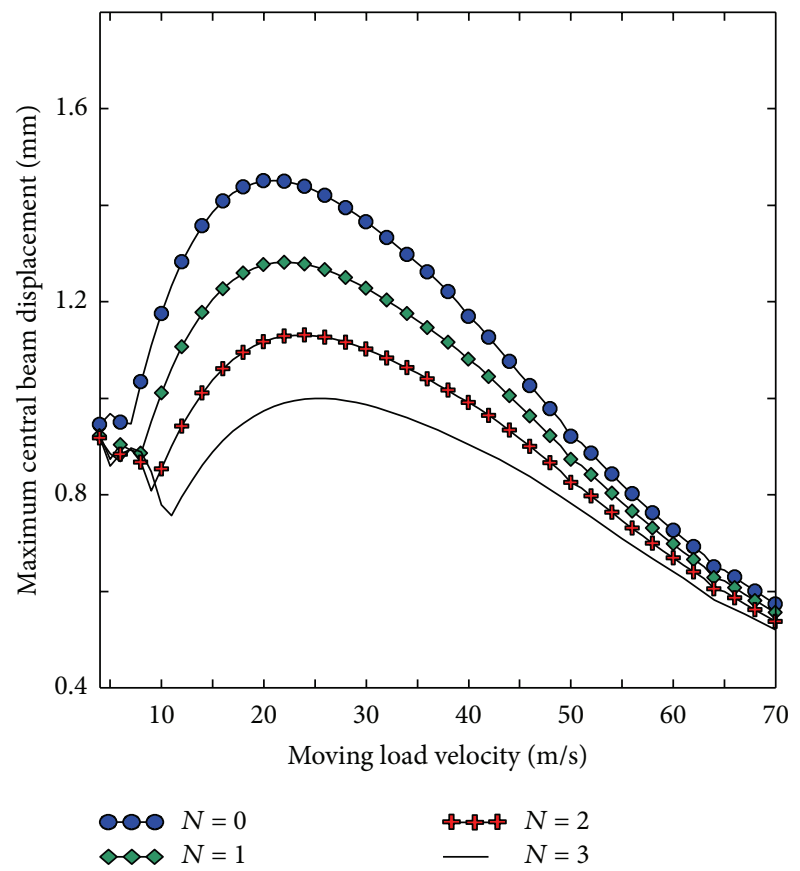

FIGURE 4: The maximum displacement of the beam at mid-span versus the velocity of the moving load for $\zeta=0.075$.

a two-SDOF system, while in [50], it was analyzed using the Euler-Bernoulli theory. In [50], the Galerkin-Bubnov variation method is used to solve the beam problem with only one absorber attached at the mid-span of the beam. The order of the fractional derivative for both of the beam and the oscillator is assumed to be unity; that is, $\beta=\gamma=1$. 


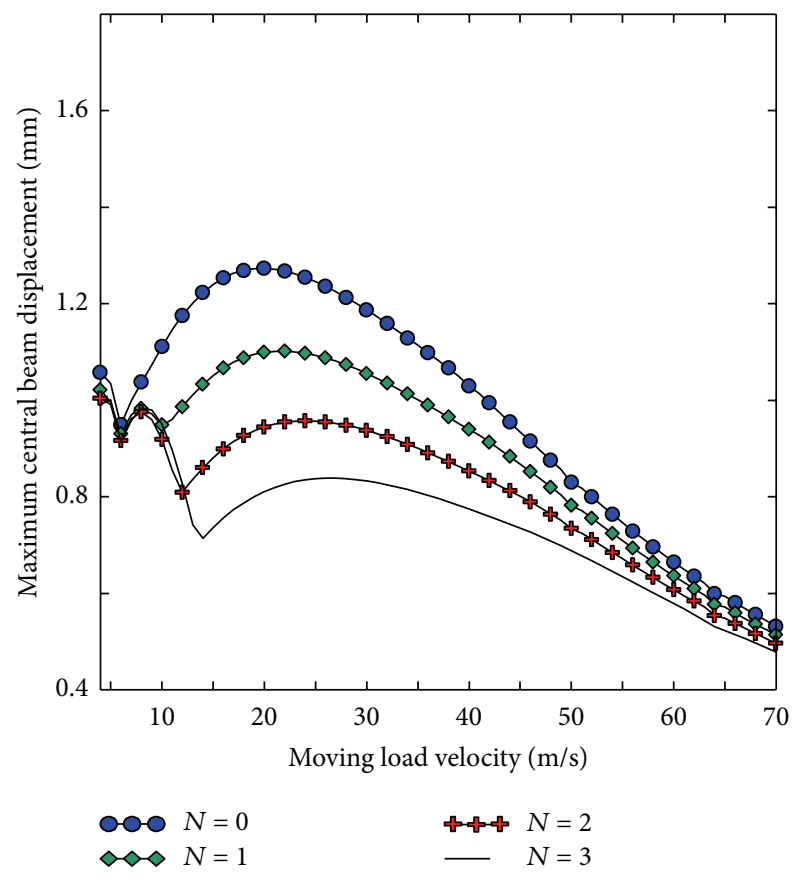

Figure 5: The maximum displacement of the beam at mid-span versus the velocity of the moving load for $\zeta=0.15$.

Figures 3-5 show the maximum response of the beam at the mid-span versus the velocity of the moving load for three different values of the beam damping ratio; that is, $\zeta=0, \zeta=0.075$, and $\zeta=0.15$, respectively. The upper two curves in Figure 3 fit precisely the published results in $[50,51]$. The curve for $(N=0)$ in Figure 3 fits exactly the well-known exact solution which is obtained by the Duhamel integral [32]. Furthermore, we note from Figures 3-5 that, as both the number of absorbers which are attached to the beam and as the damping beam-ratio increases, the dynamic response decreases. This is clearly seen from the lowest curve in Figure 5 where $N=3$ and $\zeta=0.15$.

3.3. Optimization of the Vehicle Dynamic Response. The moving load problem that was solved in Section 3.2 is solved here for the more general case of a moving vehicle $\left(k_{v}=\right.$ $700 \mathrm{~N} / \mathrm{m}, \zeta_{v}=0.1, m_{v}=1 \mathrm{~kg}$ ). Figures 6,7 , and 8 illustrate the maximum response of the beam at the mid-span versus the velocity of the moving vehicle for three different values of the damping ratio of the beam; that is, $\zeta=0, \zeta=$ 0.075 , and $\zeta=0.15$, respectively. Moreover, Figure 9 shows the maximum central beam displacement versus the velocity of the moving vehicle for different numbers of absorbers $(N=1,2)$. The curves in these figures show that the maximum central displacement of the beam decreases as the number of absorbers, beam damping ratio, and vehicle velocity increase. By comparing Figures 3-5 obtained for a moving load with Figures 6-8 obtained for a moving vehicle, it is clearly seen that the maximum displacement of the beam decreases for the moving vehicle case and this is due to the effect of the damping ratio of the vehicle. Moreover, the

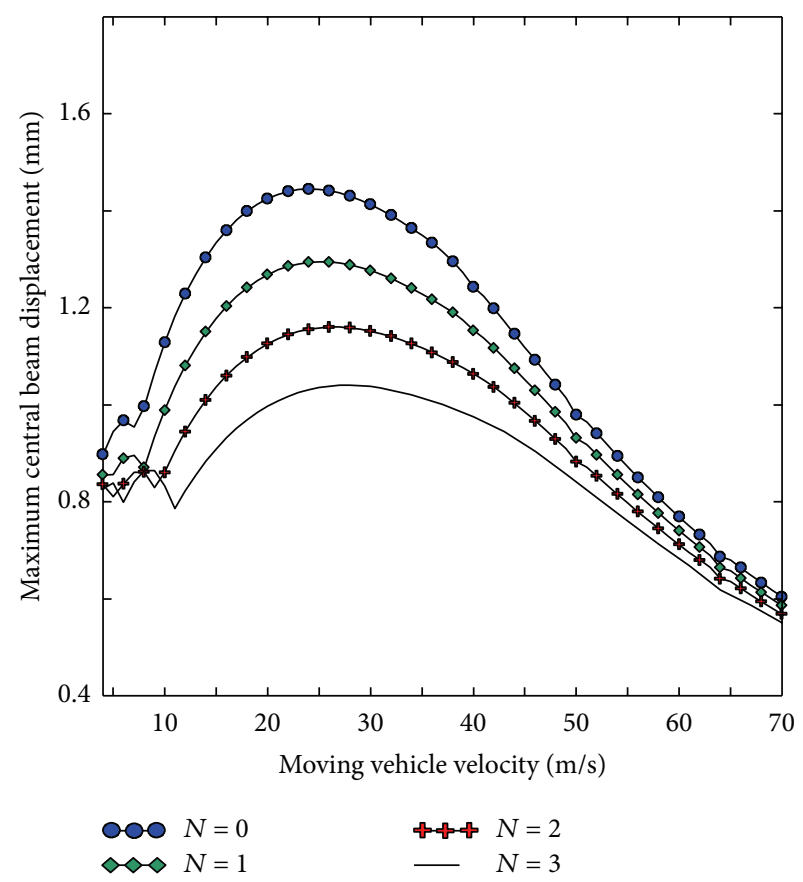

FIgURE 6: The maximum displacement of the beam at mid-span versus the velocity of the moving vehicle for $\zeta=0$.

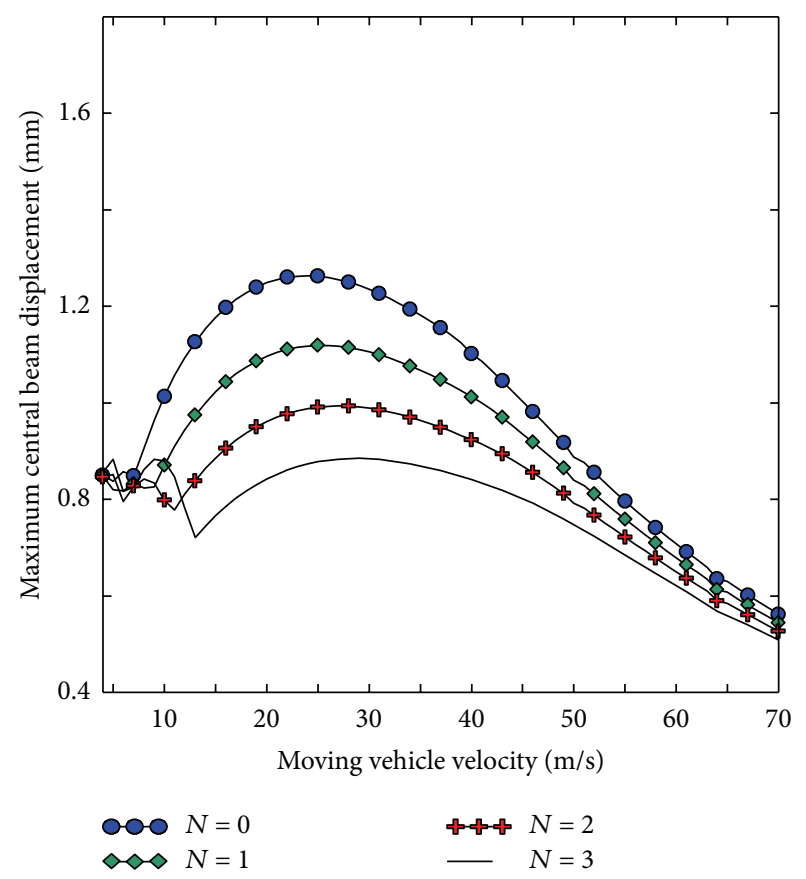

FIgURE 7: The maximum displacement of the beam at mid-span versus the velocity of the moving vehicle for $\zeta=0.075$.

critical mean velocity of the moving vehicle at which the maximum displacement occurs is $v=25 \mathrm{~m} / \mathrm{s}$, see Figures 6-8.

3.4. Optimization of the Fractional Derivatives. In this Section, one example is analyzed to show the effect of the order 


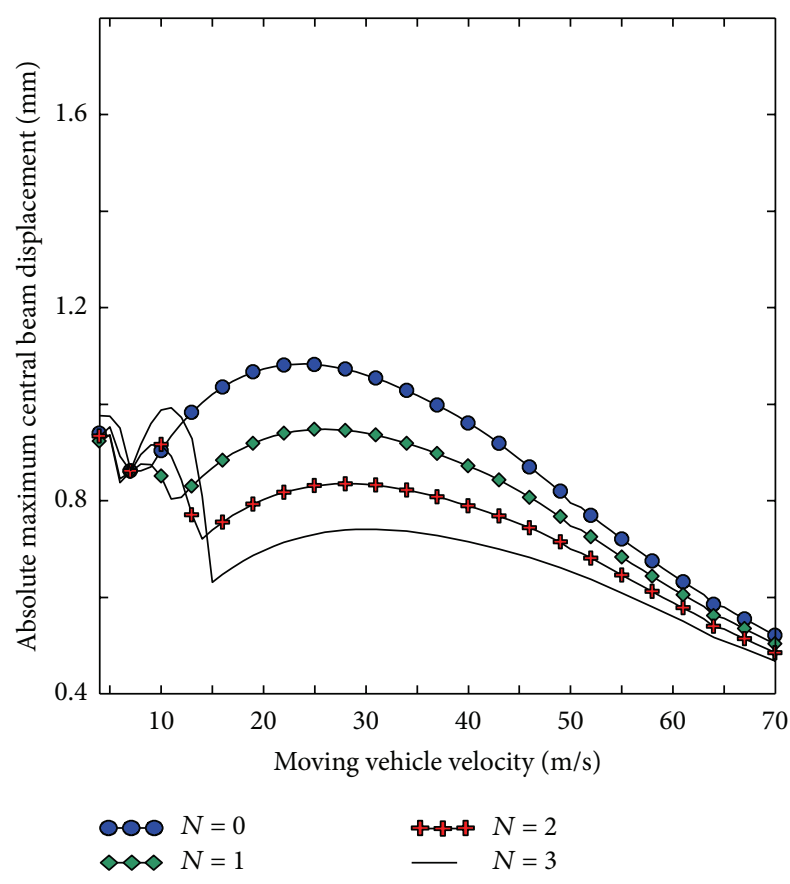

Figure 8: The maximum displacement of the beam at mid-span versus the velocity of the moving vehicle for $\zeta=0.15$.

of fractional derivatives for both of the beam and absorbers $(0<\gamma<2,0<\beta<2)$. The 3D surface shown in Figure 10 (for the data given in Table 1) represents the variation of the maximum deflection of the beam at the mid-span (where the absorber is attached) with the beam fractional derivative $(\beta)$ and the fractional derivative of the absorber $(\gamma)$. The obtained surface in Figure 10 leads to very interesting critical values of $\beta$ and $\gamma$. From this surface, the optimal values obtained are $\beta=1.245$ and $\gamma=1.190$, at which the minimum beam deflection occurs. This reduction is due to the effect of the damper added to the vehicle. The optimal values obtained from the surface shown in Figure 10 further show the physical meaning of fractional derivative, which is equivalent to increasing the damping characteristics of the beam.

3.5. Optimization of the Number of Fractionally-Damped Oscillators. In this section, the optimal values that were obtained in Sections 3.3 and $3.4(\beta=1.245, \gamma=1.19, v=$ $25 \mathrm{~m} / \mathrm{s}$ ) are used to determine the dynamic response of the beam attached to many SDOF systems. The other parameters considered in this example are shown in Table 1. Figures 11, 12, 13 , and 14 show the dynamic response of the beam for various values of beam damping ratio $(\zeta=0, \zeta=0.075, \zeta=0.15)$, respectively. The effects of the beam damping ratio and the number of oscillators are clearly observed from the curves in Figures 11-14.

The effect of the previously obtained optimal values is observed clearly when both Figures 5 and 8 are compared with Figure 13. The percent relative reduction in the maximum central deflection (when the beam subjected to a moving load is compared to that subjected to a moving

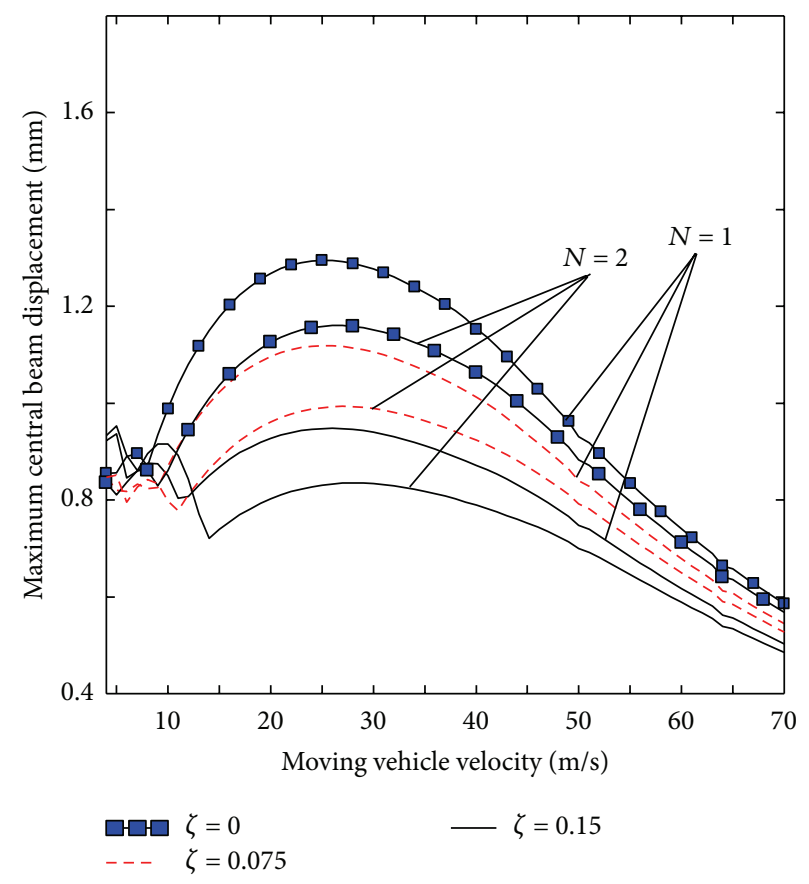

FIGURE 9: The maximum displacement of the beam at mid-span versus the velocity of the moving vehicle with one and two $(N=1,2)$ absorbers attached.

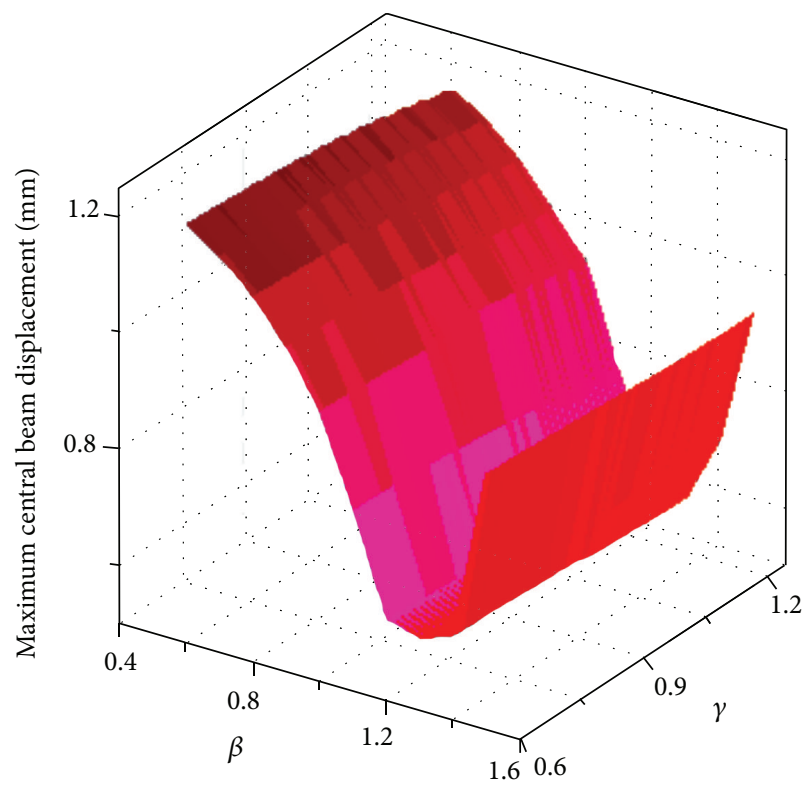

FIGURE 10: The maximum central deflection of the beam against the order of the beam's fractional derivative and the fractional derivative of the absorber $(\beta, \gamma)$.

vehicle) is computed for ( $N=0, N=1, N=2$, and $N=3)$ as $(14.5 \%, 13.85 \%, 12.74 \%$, and $11.63 \%)$, respectively, see Figures 5 and 8 , while the percent relative reduction in the maximum central deflection when Figure $8(\beta=1$ and $\gamma=1)$ is compared with Figure $13(\beta=1.245$ and $\gamma=1.19)$ of the beams subjected to a moving vehicle is computed for $(N=0$, 


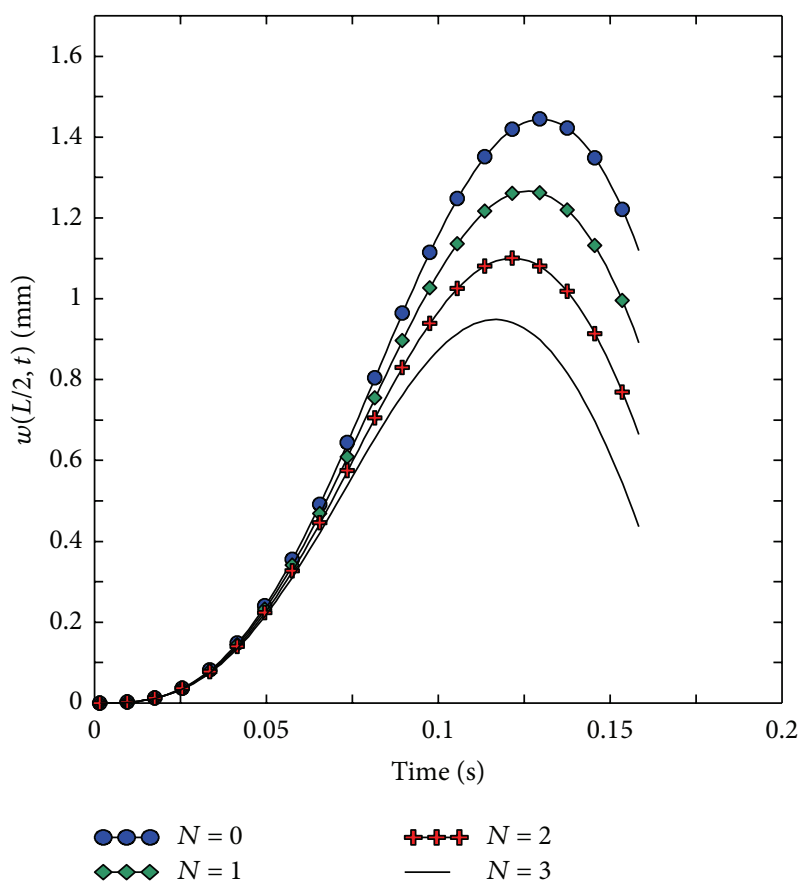

FIgURE 11: The dynamic response $(w(L / 2, t))$ for the beam with $\zeta=$ 0 , subjected to a moving vehicle and attached to many absorbers.

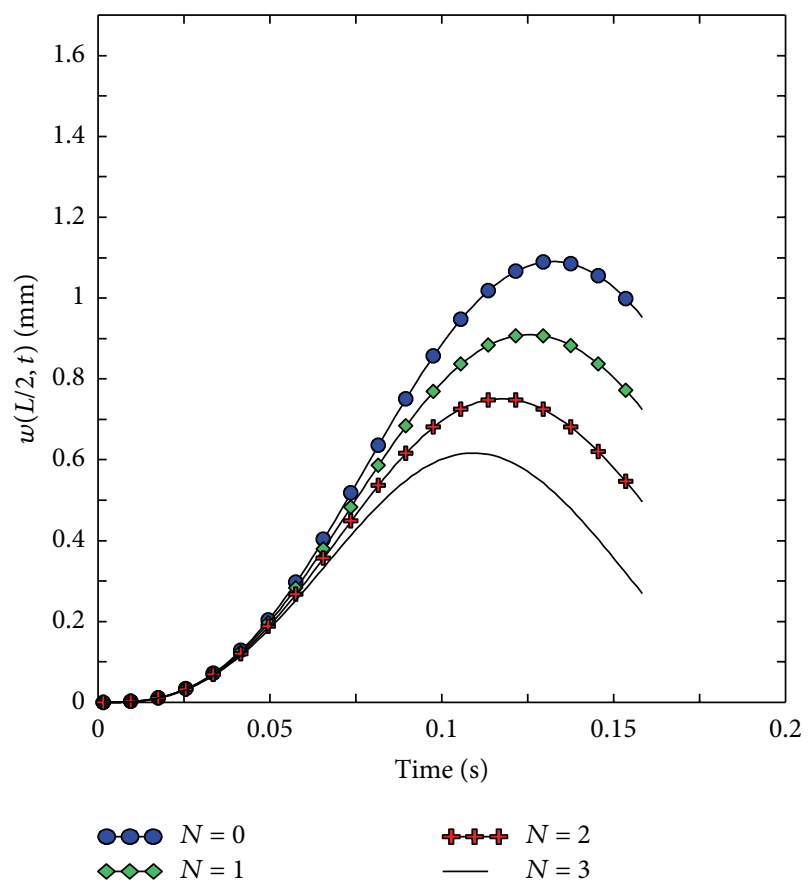

FIGURE 12: The dynamic response $(w(L / 2, t))$ for the beam with $\zeta=$ 0.075 , subjected to a moving vehicle and attached to many absorbers.

$N=1, N=2$, and $N=3)$ as $(27.58 \%, 41.27 \%, 48.99 \%$, and $54.54 \%$ ), respectively, see Figures 8 and 13 . This reduction in the dynamic response of the beam is due to the effect of the damping of the beam, fractional damping, number of absorbers, and the vehicle-damping. However, the influence of the order of fractional derivatives, beam damping ratio,

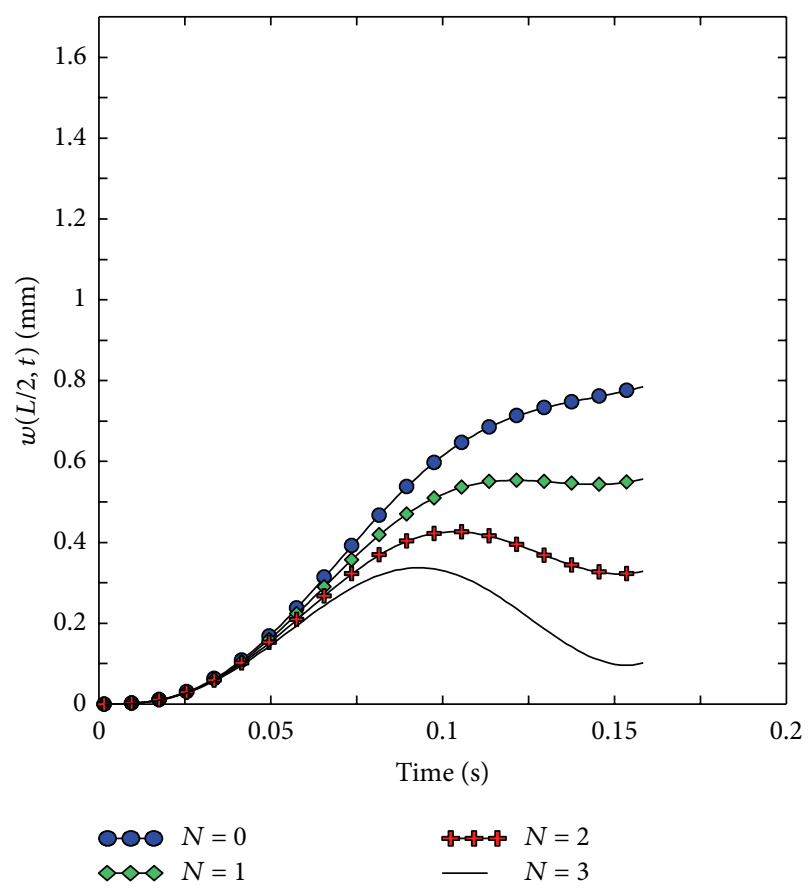

FIGURE 13: The dynamic response $(w(L / 2, t))$ for the beam with $\zeta=$ 0.15 , subjected to a moving vehicle attached to many absorbers.

oscillators damping ratios, and the number of the attached oscillators on the beam dynamic response is clearly observed.

Figure 14 shows the dynamic response of the beam versus time for both of the moving load and vehicle for the beam damping ratio $(\zeta=0.15)$ for which $(N=0, N=1, N=2$, $N=3$ ). The fractional derivatives for both the beam and absorbers are taken as $\beta=1.245$ and $\gamma=1.19$, respectively. The effect of these optimal values on the beam response is clearly observed. The reduction in the dynamic response of the beam is due to the effect of the damping of the beam, fractional damping, number of attached absorbers, and the vehicle-damping.

\section{Conclusions and Future Work}

In this paper, an analytical model is introduced to solve the transverse vibration of Bernoulli-Euler beam attached to N-SDOF absorbers with damping characteristics that are described in terms of fractional derivatives. The damping characteristics of the beam and absorbers are assumed to be of fractional-order derivatives. The method employed uses the Laplace transform with decomposing the displacement into infinite series to find the solution. However, the solution may be written in a closed form for some special cases; otherwise, it may be truncated and produced by using appropriate mathematical software such as Maple.

The numerical results obtained in this paper, illustrate that (1) the dynamic response decreases as the number of absorbers attached to the beam increases, (2) as both the damping-ratios of absorbers and beam increase, the dynamic response decreases, (3) the type of load further affects the 


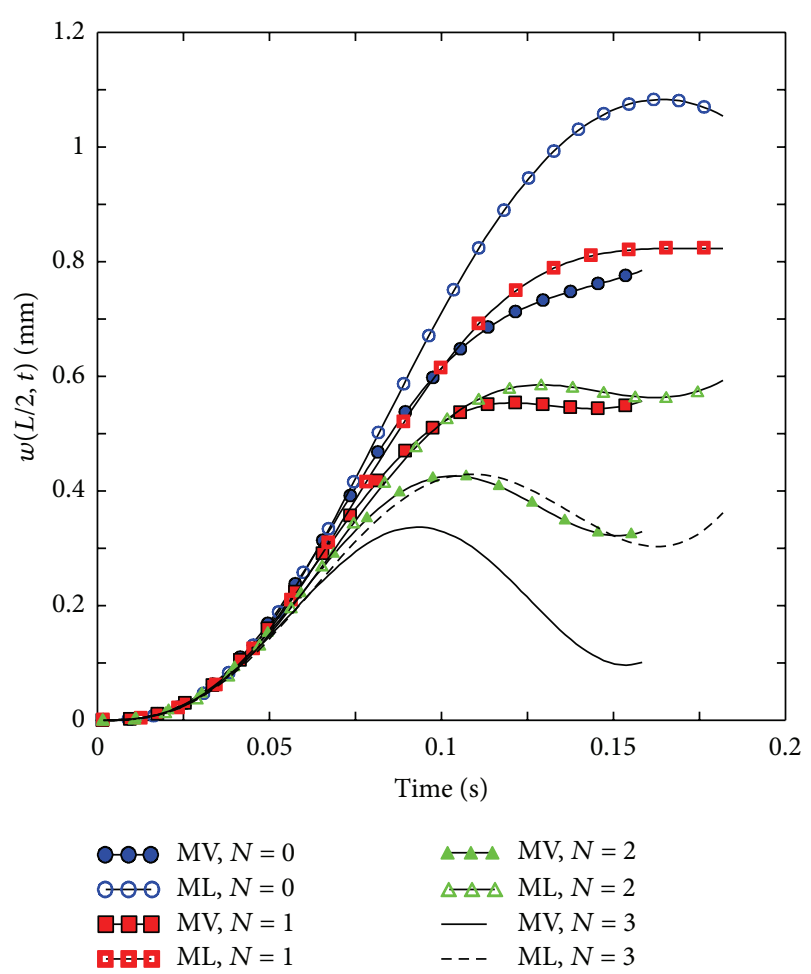

FIgURE 14: The dynamic response $(w(L / 2, t))$ for the beam with $\zeta=0.15$, attached to many absorbers for moving vehicle (MV) and moving load (ML).

dynamic response of the beam; hence, a moving vehicle reduces the response due to its damping property, and (4) there are some critical values of $\beta$ and $\gamma$ at which the beam has less deflection than that which is obtained from the fullorder derivative models (i.e., $\beta=1$ and $\gamma=1$ ).

Finally, even though the fractional derivative model provides better damping models of fractionally-damped structures and materials, a limited number of papers have been presented in this field. This is probably due to the fact that the underlying mathematics of the fractional derivative models are sophisticated and not well-developed. Hence, developments in this field will lead to the development of the applications of fractional derivative models in several other engineering problems.

The approach of the fractional derivative damping, used in this paper, will allow researchers to choose suitable mathematical models that may better converge to realistic experimental models than the well-known conventional firstorder damping models. Hence, the fractional derivative models may precisely represent a nonlinear damping behavior better than other existing damping models.

Future work will involve the following items for the application of the fractional calculus-based approach that is used in this paper within: (1) quantum and nano computing systems [52], (2) nanobeams, materials and medical applications [52-54], (3) fractal-based formulations to the microquasistatic thermoviscoelastic creep for rough surfaces in contact [55], and (4) other beam configurations for various types of nonlinearities, damping characteristics, and moving vehicles.

\section{References}

[1] R. L. Bagley and P. J. Torvik, "On the appearance of the fractional derivative in the behavior of real materials," Journal of Applied Mechanics, vol. 51, no. 2, pp. 294-298, 1984.

[2] R. L. Bagley and P. J. Torvik, "On the fractional calculus model of viscoelastic behavior," Journal of Rheology, vol. 30, no. 1, pp. 133-155, 1986.

[3] E. G. Bakhoum and C. Toma, "Transient aspects of wave propagation connected with spatial coherence," Mathematical Problems in Engineering, vol. 2013, Article ID 691257, 5 pages, 2013.

[4] C. Koh and J. M. Kelly, "Application of fractional derivatives to seismic analysis of base- isolated models," Earthquake Engineering \& Structural Dynamics, vol. 19, no. 2, pp. 229-241, 1990.

[5] N. Makris and M. C. Constantinou, "Fractional-derivative Maxwell model for viscous dampers," Journal of Structural Engineering, vol. 117, no. 9, pp. 2708-2724, 1991.

[6] T. Pritz, "Five-parameter fractional derivative model for polymeric damping materials," Journal of Sound and Vibration, vol. 265, no. 5, pp. 935-952, 2003.

[7] H. Qi and M. Xu, "Unsteady flow of viscoelastic fluid with fractional Maxwell model in a channel," Mechanics Research Communications, vol. 34, no. 2, pp. 210-212, 2007.

[8] N. Shimizu and W. Zhang, "Fractional calculus approach to dynamic problems of viscoelastic materials," JSME International Journal C, vol. 42, no. 4, pp. 825-837, 1999.

[9] M. Li, S. C. Lim, and S. Y. Chen, "Exact solution of impulse response to a class of fractional oscillators and its stability," Mathematical Problems in Engineering, vol. 2011, Article ID 657839, 9 pages, 2011.

[10] M. Li, C. Cattani, and S. Y. Chen, "Viewing sea level by a onedimensional random function with long memory," Mathematical Problems in Engineering, vol. 2011, Article ID 654284, 13 pages, 2011.

[11] M. Li, "Fractal time series: a tutorial review," Mathematical Problems in Engineering, vol. 2010, Article ID 157264, 26 pages, 2010.

[12] M. Li and W. Zhao, "Representation of a stochastic traffic bound," IEEE Transactions on Parallel and Distributed Systems, vol. 21, no. 9, pp. 1368-1372, 2010.

[13] M. Li, "Approximating ideal filters by systems of fractional order," Computational and Mathematical Methods in Medicine, vol. 2012, Article ID 365054, 6 pages, 2012.

[14] M. Li, Y. Q. Chen, J. Y. Li, and W. Zhao, "Hölder scales of sea level," Mathematical Problems in Engineering, vol. 2012, Article ID 863707, 22 pages, 2012.

[15] M. Li, S. C. Lim, C. Cattani, and M. Scalia, "Characteristic roots of a class of fractional oscillators," Advances in High Energy Physics, vol. 2013, Article ID 853925, 7 pages, 2013.

[16] C. Toma, "Wavelets-computational aspects of sterian-realistic approach to uncertainty principle in high energy physics-A transient approach," Advances in High Energy Physics, vol. 2013, Article ID 735452, 6 pages, 2013.

[17] M. Li and W. Zhao, "On 1/f noise," Mathematical Problems in Engineering, vol. 2012, Article ID 673648, 23 pages, 2012.

[18] M. Li and W. Zhao, "Visiting power laws in cyber-physical networking systems," Mathematical Problems in Engineering, vol. 2012, Article ID 302786, 13 pages, 2012.

[19] M. Li, W. Zhao, and C. Cattani, "Delay bound: fractal traffic passes through servers," Mathematical Problems in Engineering, vol. 2013, Article ID 157636, 15 pages, 2013. 
[20] H. Sheng, Y. Q. Chen, and T. S. Qiu, Fractional Processes and Fractional Order Signal Processing, Springer, 2012.

[21] R. Abu-Mallouh, I. Abu-Alshaikh, H. S. Zibdeh, and K. Ramadan, "Response of fractionally damped beams with general boundary conditions subjected to moving loads," Shock and Vibration, vol. 19, no. 3, pp. 333-347, 2012.

[22] B. N. N. Achar, J. W. Hanneken, and T. Clarke, "Damping characteristics of a fractional oscillator," Physica A, vol. 339, no. 3-4, pp. 311-319, 2004.

[23] O. P. Agrawal, "Analytical solution for stochastic response of a fractionally damped beam," Journal of Vibration and Acoustics, vol. 126, no. 4, pp. 561-566, 2004.

[24] S. S. Ray, B. P. Poddar, and R. K. Bera, "Analytical solution of a dynamic system containing fractional derivative of order onehalf by adomian decomposition method," Journal of Applied Mechanics, vol. 72, no. 2, pp. 290-295, 2005.

[25] K. S. Fa, "A falling body problem through the air in view of the fractional derivative approach," Physica A, vol. 350, no. 2-4, pp. 199-206, 2005.

[26] A. Chatterjee, "Statistical origins of fractional derivatives in viscoelasticity," Journal of Sound and Vibration, vol. 284, no. 3-5, pp. 1239-1245, 2005.

[27] E. Goldfain, "Fractional dynamics and the Standard Model for particle physics," Communications in Nonlinear Science and Numerical Simulation, vol. 13, no. 7, pp. 1397-1404, 2008.

[28] A. Hernández-Jiménez, J. Hernández-Santiago, A. MaciasGarcía, and J. Sánchez-González, "Relaxation modulus in PMMA and PTFE fitting by fractional Maxwell model," Polymer Testing, vol. 21, no. 3, pp. 325-331, 2002.

[29] E. Savin, "Dynamic amplification factor and response spectrum for the evaluation of vibrations of beams under successive moving loads," Journal of Sound and Vibration, vol. 248, no. 2, pp. 267-288, 2001.

[30] Y. B. Yang and C. W. Lin, "Vehicle-bridge interaction dynamics and potential applications," Journal of Sound and Vibration, vol. 284, no. 1-2, pp. 205-226, 2005.

[31] H. S. Zibdeh and R. Rackwitz, "Moving loads on beams with general boundary conditions," Journal of Sound and Vibration, vol. 195, no. 1, pp. 85-102, 1996.

[32] L. Fryba, Vibration of Solids and Structures under Moving Loads, Noordhoff International Publishing, Groningen, The Netherlands, 1972.

[33] Y. Chen, C. A. Tan, and L. A. Bergman, "Effects of boundary flexibility on the vibration of a continuum with a moving oscillator," Journal of Vibration and Acoustics, Transactions of the ASME, vol. 124, no. 4, pp. 552-560, 2002.

[34] M. Olsson, "On the fundamental moving load problem," Journal of Sound and Vibration, vol. 145, no. 2, pp. 299-307, 1991.

[35] M. A. Foda and Z. Abduljabbar, "A dynamic green function formulation for the response of a beam structure to a moving mass," Journal of Sound and Vibration, vol. 210, no. 3, pp. 295306, 1998.

[36] H. P. Lee, "Dynamic response of a beam with a moving mass," Journal of Sound and Vibration, vol. 191, no. 2, pp. 289-294, 1996.

[37] G. Michaltsos, D. Sophianopoulos, and A. N. Kounadis, "The effect of a moving mass and other parameters on the dynamic response of a simply supported beam," Journal of Sound and Vibration, vol. 191, no. 3, pp. 357-362, 1996.

[38] S. Sadiku and H. H. E. Leipholz, "On the dynamics of elastic systems with moving concentrated masses," Ingenieur-Archiv, vol. 57 , no. 3, pp. 223-242, 1987.
[39] A. V. Pesterev and L. A. Bergman, "An improved series expansion of the solution to the moving oscillator problem," Journal of Vibration and Acoustics, vol. 122, no. 1, pp. 54-61, 2000.

[40] B. Yang, C. A. Tan, and L. A. Bergman, "Direct numerical procedure for solution of moving oscillator problems," Journal of Engineering Mechanics, vol. 126, no. 6, pp. 462-469, 2000.

[41] Y. B. Yang and Y. S. Wu, "A versatile element for analyzing vehicle-bridge interaction response," Engineering Structures, vol. 23, pp. 452-469, 2001.

[42] F. Samani, F. Pellicano, and A. Masoumi, "Performance of nonlinear vibration absorbers for beams under moving vehicles," ENOC 2011, Rome, Italy, July 2011.

[43] D. Stăncioiu, H. Ouyang, and J. E. Mottershead, "Vibration of a beam excited by a moving oscillator considering separation and reattachment," Journal of Sound and Vibration, vol. 310, no. 4-5, pp. 1128-1140, 2008.

[44] J. W. Nicholson and L. A. Bergman, "Free vibration of combined dynamical systems," ASCE Journal of Engineering Mechanics, vol. 112, no. 1, pp. 1-13, 1986.

[45] Z.-F. Liang and X.-Y. Tang, "Analytical solution of fractionally damped beam by Adomian decomposition method," Applied Mathematics and Mechanics, vol. 28, no. 2, pp. 219-228, 2007.

[46] M. A. Hilal and H. S. Zibdeh, "Vibration analysis of beams with general boundary conditions traversed by a moving force," Journal of Sound and Vibration, vol. 229, no. 2, pp. 377-388, 2000.

[47] I. Podlubny, Fractional Differential Equations, Academic Press, San Diego, Calif, USA, 1999.

[48] Y. L. Luke, The Special Functions and Their Approximations, Academic Press, New York, NY, USA, 1969.

[49] G. Venkatesan, Dynamic behavior of bridge structure under moving load [Ph.D. thesis], University of Oklahoma, 2009.

[50] F. S. Samani and F. Pellicano, "Vibration reduction on beams subjected to moving loads using linear and nonlinear dynamic absorbers," Journal of Sound and Vibration, vol. 325, no. 4-5, pp. 742-754, 2009.

[51] J.-J. Wu, "Study on the inertia effect of helical spring of the absorber on suppressing the dynamic responses of a beam subjected to a moving load," Journal of Sound and Vibration, vol. 297, no. 3-5, pp. 981-999, 2006.

[52] A. N. Al-Rabadi, "Carbon nanotube (CNT) multiplexers, circuits, and actuators," United States Patent and Trademark Office (USPTO), Patent no. US 7, 508, 039 B2, March 2009.

[53] C. Cattani and A. Ciancio, "Separable transition density in the hybrid model for tumor-immune system competition," Computational and Mathematical Methods in Medicine, vol. 2012, Article ID 610124, 6 pages, 2012.

[54] C. Cattani, A. Ciancio, and B. Lods, "On a mathematical model of immune competition," Applied Mathematics Letters, vol. 19, no. 7, pp. 678-683, 2006.

[55] O. M. Abuzeid, A. N. Al-Rabadi, and H. S. Alkhaldi, "Recent advancements in fractal geometric-based nonlinear time series solutions to the micro-quasistatic thermoviscoelastic creep for rough surfaces in contact," Mathematical Problems in Engineering, vol. 2011, Article ID 691270, 29 pages, 2011. 


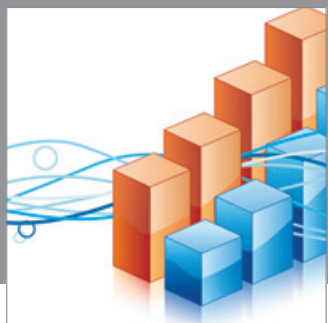

Advances in

Operations Research

mansans

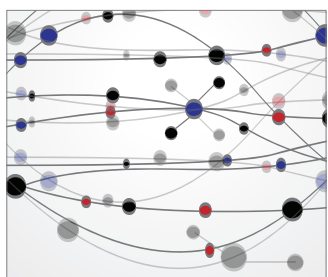

The Scientific World Journal
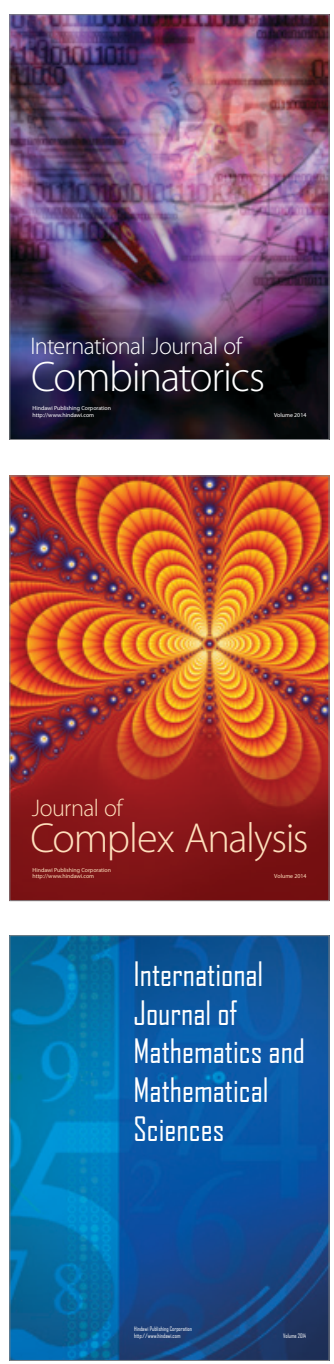
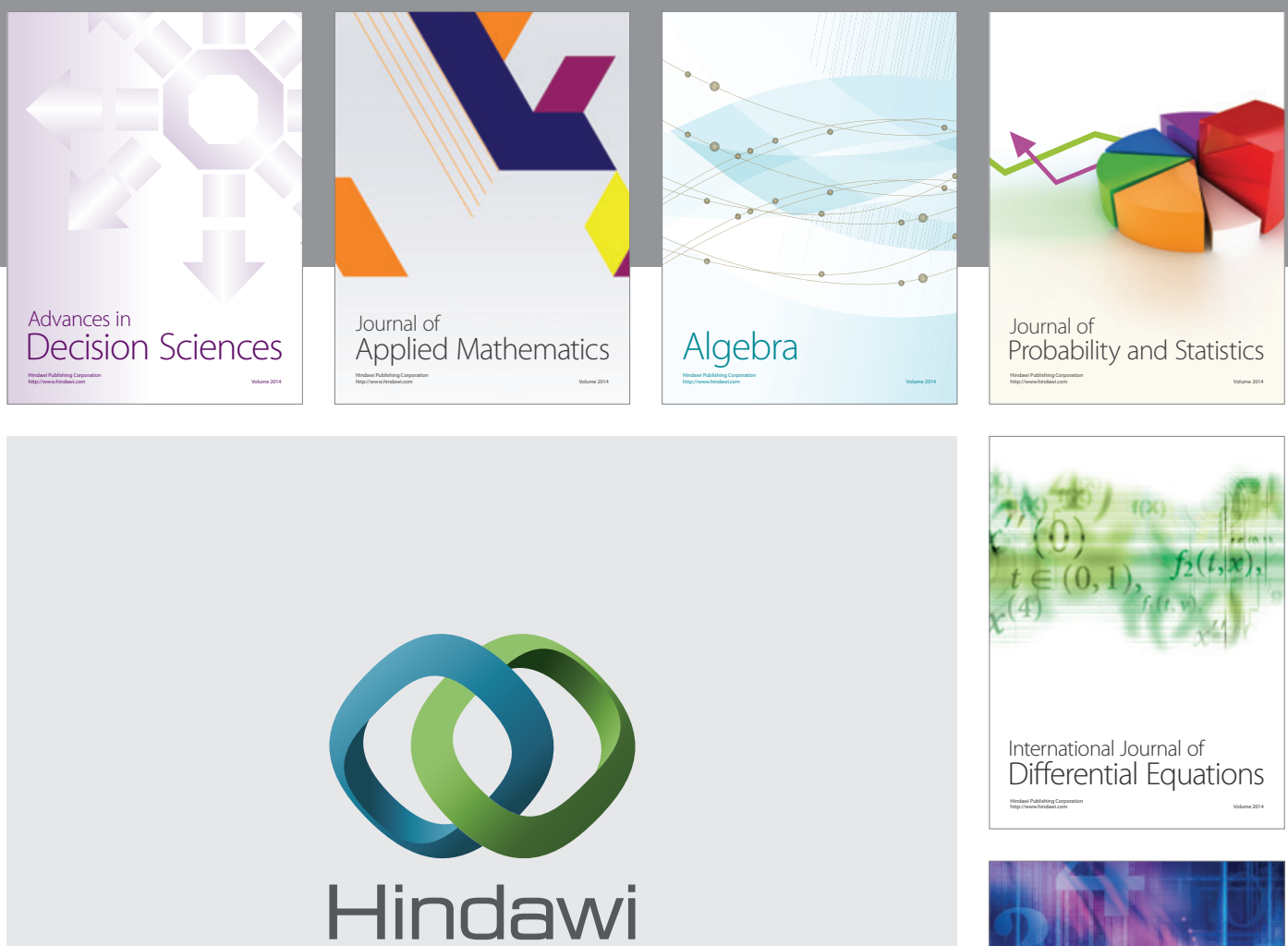

Submit your manuscripts at http://www.hindawi.com
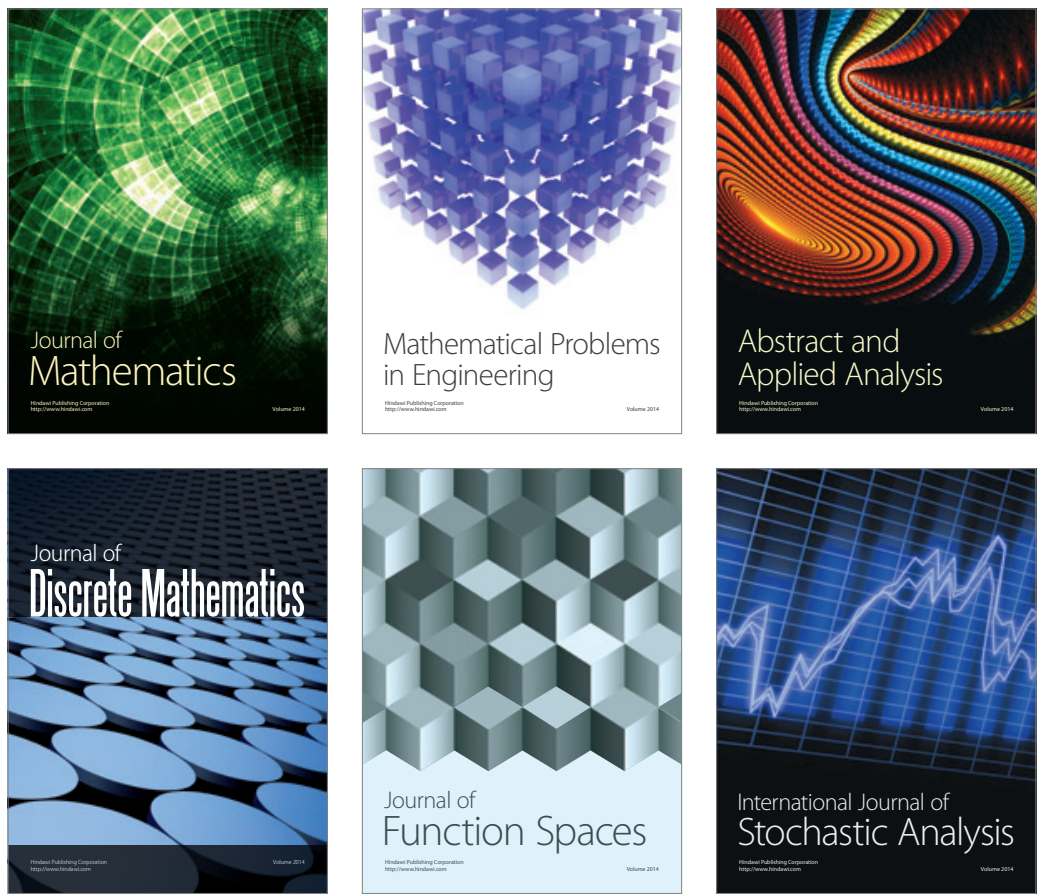

Journal of

Function Spaces

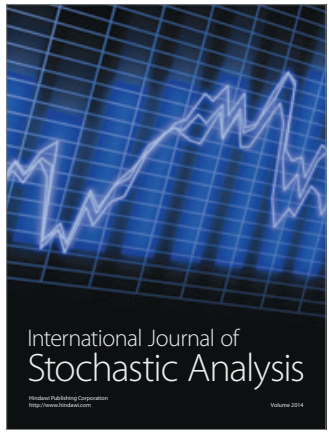

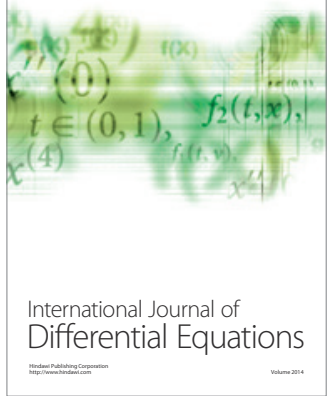
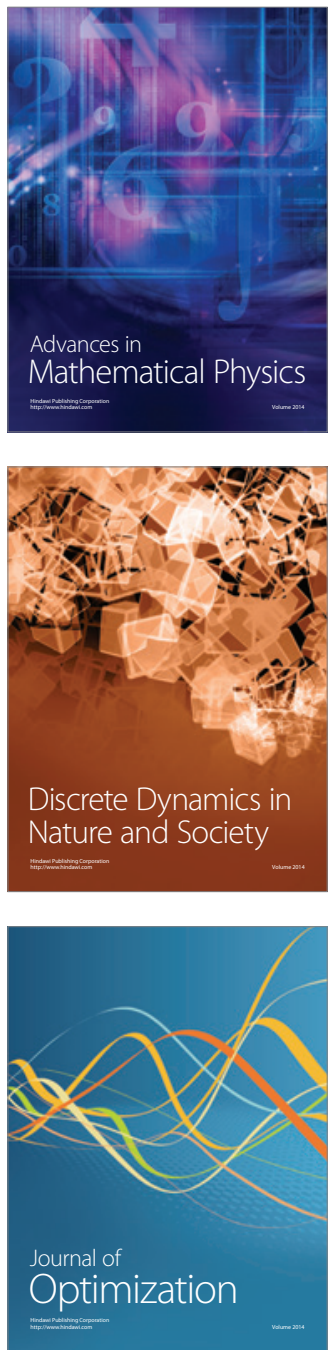